\title{
Quality of Partogram Recordings and Perinatal Outcome at Muhimbili National Hospital Tanzania
}

\author{
Paschal Francis Mdoe ${ }^{1 *}$, Prisca Jonas Kaminsa ${ }^{2}$ and Siriel Massawe ${ }^{1}$ \\ ${ }^{1}$ Muhimbili University of Health and Allied Sciences, Tanzania \\ ${ }^{2}$ Tanzania Ministry of Health, Tanzania
}

*Corresponding author: Paschal Francis Mdoe, Muhimbili University of Health and Allied Sciences, Tanzania, Tel: +255754429346; Email: pfmdoe@gmail.com

\author{
Research Article \\ Volume 2 Issue 2 \\ Received Date: May 07, 2018 \\ Published Date: May 21, 2018 \\ DOI: $10.23880 /$ whsj-16000114
}

\section{Abstract}

Background: The management of labor using partograph is the standard way of improving both maternal and perinatal outcomes. The partogram increases the quality and regularity of all observations on the fetus and the mother in labor and aids early recognition of problems in both mother and fetus. One third of stillbirths takes place during delivery and is largely avoidable.

Aim: To assess the quality of partogram recordings and compare with fetal outcomes among women delivering at Muhimbili National Hospital.

Methods: A cross-sectional study was conducted from 1st September to 30th November 2011. Partographs of women delivered at the hospital were reviewed immediately after delivery. Using checklist recordings of parameters, the progress of labour, fetal outcome (Apgar score at 5 minutes, still birth and admission to neonatal ward for special care) and immediate maternal outcomes (PPH, perineal tear, visceral injuries following cesarean section) were assessed. Partograph recordings were judged as standard if standard as per standard protocols.

Results: Partograph was used for all women admitted with spontaneous labor. 1,051 partographs were reviewed during the study time. Majority (79.5\%) had spontaneous vaginal delivery and $18.6 \%$ delivered by cesarean section. Only $8.9 \%$ of partographs had all parameters with standard recordings. The most unrecorded parameter was maternal pulse rate. Most (81.4\%) of partographs had substandard recording of uterine contractions.

There were 53 newborns with Apgar score less than 7 at 5 minutes and 17 fresh stillbirths. Substandard fetal heart rate recording was significantly associated with Apgar score less than 7 at 5 minute and with stillbirths (P-value $<0.001$, Chisquare 15.92).

Conclusion: This study as other studies conducted as where found high proportion of substandard partogram recordings. Supportive supervision and on job training on correct use of partograph is recommended as a way of improving labor monitoring and improved fetal and maternal outcomes.

Keywords: Partogram; Labor; Perinatal outcomes 


\section{Women's Health Science Journal}

Abbreviations: BP: Blood Pressure; CS: Cesarean Section; FHR: Fetal Heart Rate; IPPM: Intramural private practice Muhimbili; PPH: Post-partum Hemorrhage; PR: Pulse Rate; MUHAS: Muhimbili University of Health and Allied Sciences; MNH: Muhimbili National Hospital; OR: Odds Ratio; SPSS: Statistical Package of Social Sciences.

\section{Introduction}

\section{Background}

Labor management using partogram is the standard way of insuring better perinatal and maternal outcomes in resource limited settings $[1,2]$. The partogram is a tool that serves as an early warning system and can assist in early decision making on transfer, augmentation or immediate delivery [3]. The partogram increases the quality and regularity of all observations on the fetus and the mother in labor and aids early recognition of problems in both maternal and baby [1].

The partogram has three components, which includes the fetal condition, the progress of labor and the maternal condition. One of the parameters used in monitoring of fetal condition during labor include fetal heart rate, according to WHO protocol is recorded half hourly in first stage of labor and every 15 minutes in second stage of labor. It is judged to be standard if at least recorded hourly [4].

Other parameters are membrane status, liquor quality and amount, and molding. The progress of labor is central part of partogram, records the rate of cervical dilatation, descent of presenting part and pattern and strength of uterine contractions. The third component record maternal temperature, pulse rate, maternal blood pressure, and regular urinalysis [4].

Partogram has been recommended by the WHO for monitoring progress of labor world-wide. The aim of partogram recommendation is to improve maternal and perinatal outcome. Despite the recommendation of WHO on partogram use, the perinatal mortality is still high especially in developing countries [5]. One third of stillbirths takes place during delivery and is largely avoidable [6]. This high perinatal mortality rate has been associated with substandard partogram recordings of fetal heart rates which may be reflecting substandard monitoring of labor $[7,8]$. Lack of partogram use during labor and less than one FHR assessment per hour during labor were significantly associated with perinatal deaths [9].
Perinatal outcome in terms of Apgar score $<7$ at 5 minutes has been associated with majority of neonatal deaths [8]. Adverse neonatal outcomes in terms of 5 minutes Apgar score $<7$, early neonatal deaths and intrapartum stillbirth are not only related to maternal health but also it is the best indicator for the quality of health care services [10]. This study aimed at assessing the partogram recordings if they are done as per standard and whether the recordings have effect on fetal outcomes in terms of Apgarscore at 5 minutes, stillbirth and admission to the neonatal ward.

\section{Methods}

A cross sectional observation study was conducted to review partograms of women post-delivery from $1^{\text {st }}$ September to $30^{\text {th }}$ November 2011 at Muhimbili National hospital.

Muhimbili National hospital is a tertiary referral and a teaching hospital for the Muhimbili University of Health and Allied Sciences (MUHAS). The maternity block consists of seven wards, four antenatal and postnatal wards. Each ward has 38 beds capacity used to admit antenatal and postnatal mothers. Other wards are labor ward with 20 delivery beds and postnatal ward for observation of mothers after uncomplicated normal delivery before they are discharged home. The remaining ward is used as an obstetric intensive care unit for the patients with eclampsia and mothers in need of critical care.

There is a special neonatal care unit which provides neonatal care to premature newborns and sick babies born at MNH and surrounding hospitals. Babies born by CS are directly admitted at least for one day in neonatal unit for care or till their mothers become ambulant.

MNH offers specialized obstetric services for Dar es salaam city and its suburbs, through its department of obstetrics and gynecology. The city of Dar es salaam is estimated to have a population of more than four million people. Patients are also referred from all 3 districts in city and nearby Coast region. Apart from the referred patients, a substantial number of patients with or without obstetric complication come directly from home.

Delivery data, progress of labor, maternal and neonatal outcome are recorded in the partogram. All these informations are finally summarized in midwifery book before entering them into the obstetric data base. 


\section{Women's Health Science Journal}

\section{Study Population and Sampling}

All Partograms of women delivered at MNH during the study time were included in the study.

\section{Exclusion criteria}

Women with the following characteristics their partograms were excluded from the study

- Admitted with cervical dilatation $>8 \mathrm{~cm}$

- Antepartum hemorrhage

- Multiple pregnancy

- Premature labor (Labor before 37 completed weeks)

- Preeclampsia/eclampsia

- Malpresentations (Non-cephalic presentation)

- Previous uterine scar

- Admitted for elective cesarean section and IPPM patients

- Premature rupture of membrane (Rupture of membrane more than 6 hours before onset of labor)

- IUFD and those admitted with fetal distress.

\section{Sample Size}

We considered in this study, partograms of all pregnant women, who were admitted to delivery in the hospital during the months of September, October and November 2011 and whom labor was monitored with partogram from active phase. Those with exclusion criteria their partograms were not reviewed.

\section{Data Collection}

Data were collected by using a checklist. All files of women soon post-delivery were reviewed and the information from partograms was collected using a checklist. The inpatient numbers from the file were recorded in the checklist to avoid repetitions.

The checklist with 27 items grouped into 4 sections were used for extracting information from the partogram. The first part recorded information about patient's age, gravidity, parity, estimated gestation age (by dates) and estimated time between onset of labor and hospital admission.

Second part recorded the parameters of labor which were fetal heart rate, liquor state, molding/caput, cervical dilatation, descent of head, uterine contraction, maternal $\mathrm{BP}$, maternal temperature, maternal PR and maternal urine analysis. Each parameter was assessed as not recorded, standard or substandard recorded according to protocol of standard care (Operational definition).
The third part recorded the fetal outcomes. Fetal outcomes were assessed in terms of live birth (Apgar score at 1 and 5 minutes) still birth, need of resuscitation, admission to neonatal ward for special care and the reasons of admission.

The fourth part included information of the mode of delivery (spontaneous vaginal delivery, assisted vaginal delivery (when ventouse used) cesarean section and hysterectomy) and immediate maternal outcomes, recorded as good (live birth and no complication) and adverse if the woman got PPH, perineal tear (second degree and above), need of blood transfusion, urinary bladder injury, hysterectomy or bowel injury.

\section{Data Analysis}

Data was entered into and analyzed using the SPSS database program version 20.

Univariate and bivariate analysis were done, univariate analysis for frequency computations and bivariate analysis in computing associations between variables. The Chi-square test was used to measure the strength of associations between variables, a p-value of $<0.05$ was considered to be statistically significant.

\section{Ethical Clearance}

Ethical clearance was obtained from MUHAS ethical committee and permission to conduct the study was obtained from director of surgical services of Muhimbili national hospital.

All the information obtained from the partogram remained confidential. Patient name were not used. Immediate after extracting the required information from the file, the file was returned back to the authority.

\section{Operational definitions}

Adverse fetal outcomes include those newborns with Apgar score below 7 at 5 minutes, Stillbirth and admission to the neonatal ward for special care.

Immediate maternal outcome was considered good if there was no complication and adverse outcome if it was associated with complications like perineal tears (second, third and fourth degree tear), PPH, need of blood transfusion, urinary bladder injury, hysterectomy or bowel injury.

Protocols of standard care.

- Fatal heart monitored at least hourly is considered Standard 
- Descent of presenting part recorded at least four hourly is considered standard

- Cervical dilatation recorded at least four hourly is considered standard

- Uterine contractions recorded at least hourly is considered Standard

- Maternal blood pressure recorded at least four hourly is considered Standard

- Maternal pulse rate monitored at least hourly is considered Standard

If the parameter is not recorded at all is marked as No records and if recorded but not according to standard as above is marked as substandard.

A partogram with all 6 parameters with standard recordings was judged as standard and if at least one parameter with substandard recording was judged as substandard.

\section{Results}

During the study period there were 2,372 births at the hospital. Among these deliveries 716 received IPPM services and 1656 were admitted through the normal track. After reviewing the files of those who delivered through the normal track, 1051 partograms met the inclusion criteria, therefore reviewed (Figure 1).

Time between labor onset and hospital admission ranged between 1 hour and 23 hours. Majority (55.6\%) got to the hospital within 5 hours after onset of labor and less than $1 \%$ got to the hospital more than 20 hours after the onset of labor. The median time between labor onset and hospital admission were 5hours

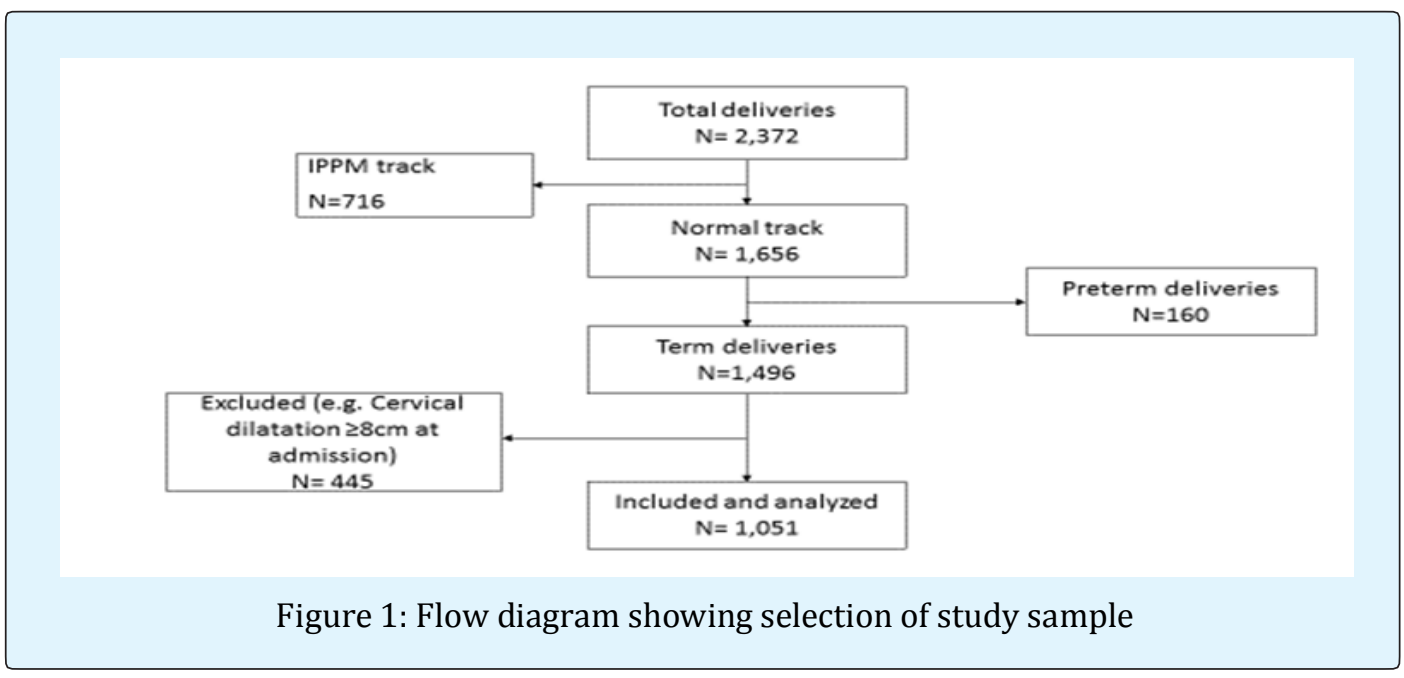

(Table 1) show that, the age bracket of 15 to 19 years shows a little predominance with $8.7 \%$ of cases. Majority
$(60.2 \%)$ of women were in the age group of 20 to 30 years, the median age was 28 (SD + 5.89 SD) years.

\begin{tabular}{|c|c|c|}
\hline & Count (N=1051) & Percentage \\
\hline \multicolumn{3}{|c|}{ Age (Years) } \\
\hline$<20$ & 91 & 8.7 \\
\hline $20-30$ & 633 & 60.2 \\
\hline $31-35$ & 207 & 19.7 \\
\hline$>35$ & 120 & 11.4 \\
\hline \multicolumn{3}{|c|}{ Gravidity (Weeks) } \\
\hline Prim & 456 & 43.4 \\
\hline $4-F e b$ & 528 & 50.3 \\
\hline$>5$ & 67 & 6.4 \\
\hline \multicolumn{2}{|c|}{ Perinatal outcomes } \\
\hline Apgar score $>7$ at 5minute & 981 & 93.5 \\
\hline Apgar score $<7$ at 5 minute & 53 & 5 \\
\hline
\end{tabular}




\section{Women's Health Science Journal}

\begin{tabular}{|c|c|c|}
\hline Stillbirth fresh & 17 & 1.6 \\
\hline \multicolumn{3}{|c|}{ Mode of Delivery } \\
\hline Spontaneous vaginal delivery & 836 & 79.5 \\
\hline Assisted vaginal delivery & 20 & 1.9 \\
\hline Caesarean section & 195 & 18.6 \\
\hline \multicolumn{2}{|c|}{ Immediate maternal outcomes } \\
\hline Good outcomes & 1029 & 97.9 \\
\hline With adverse outcomes & 22 & 2.1 \\
\hline
\end{tabular}

Table1: General characteristics of women whose partogram recordings were reviewed.

The prim gravidae represent $43.4 \%$ of the sample. Half $(50.3 \%)$ of the cases were gravida 2 to gravida 4 .

Newborns with Apgar score less than 7 at 5 minutes were $53(5 \%)$ and $17(1.6 \%)$ were stillbirth. Eighty-nine of the newborns were admitted into neonatal ward for care. Reasons for admission to neonatal ward were low Apgar score 53, Meconium aspiration 8, abnormal weight 11 (birth weight $>4 \mathrm{Kg}$ ), abnormal breathing 9 and 8 of them due to congenital malformation.

Majority $79.5 \%$ had spontaneous vaginal delivery, $18.6 \%$ had cesarean section delivery.

Twenty-two women succumbed to complications due to delivery. Two had cesarean hysterectomy, 13 got PPH,
5 had perineal tear, 1 got perineal hematoma and 1 had bowel injury

Table 2 shows that in all partograms reviewed had fetal heart rate and cervical dilatation recordings with $32.3 \%$ and $55.8 \%$ having standard recordings respectively. Maternal pulse rate is the most unrecorded parameter among all. Uterine contraction is the leading parameter having substandard records (81.4\%).

Overall $8.1 \%$ (85) of the reviewed partogram were judged to be standard. This means that they had all 5 parameters (FHR, Cervical dilatation, maternal BP, maternal body temperature and maternal PR) standard recorded.

\begin{tabular}{|c|c|c|c|}
\hline \multirow{2}{*}{ Parameter of Labor } & \multicolumn{2}{|c|}{ Recorded } & \multirow{2}{*}{ Not Recorded } \\
\cline { 2 - 3 } & Standard & Substandard & \\
\hline Fetal heart rate & $339(32.3 \%)$ & $712(67.7 \%)$ & $0(0 \%)$ \\
\hline Cervical dilatation & $586(55.8 \%)$ & $465(44.2 \%)$ & $0(0 \%)$ \\
\hline Descent & $630(59.9 \%)$ & $332(31.6 \%)$ & $89(8.5 \%)$ \\
\hline Uterine contraction & $153(14.6 \%)$ & $856(81.4 \%)$ & $42(4.0 \%)$ \\
\hline Maternal BP & $248(23.6 \%)$ & $735(69.9 \%)$ & $68(6.55 \%)$ \\
\hline Maternal body Temperature & $234(22.3 \% 0$ & $731(69.6 \%)$ & $86(8.2 \%)$ \\
\hline Maternal PR & $460(43.8 \%)$ & $499(47.5 \%)$ & $92(8.8 \%)$ \\
\hline Overall partogram judged & $85(8.1 \%)$ & $966(91.9 \%)$ & $0(0 \%)$ \\
\hline
\end{tabular}

Table 2: Proportions of partograms with records of parameters of labor.

*Adverse fetal outcome here means Apgar score less than 7at 5minute and stillbirth, good outcome means live birth with Apgar score $>7$ at 5 minutes.

Table 3 shows that substandard fetal heart rate monitoring has statistical significant association with adverse (Apgar score less than 7 at 5 minutes and stillbirth.) fetal outcomes ( $\mathrm{p}$ value $=000033$, Chi-square 15.92). 


\section{Women's Health Science Journal}

\begin{tabular}{|c|c|c|c|c|}
\hline \multirow{2}{*}{ Parameter of labor } & \multicolumn{2}{|c|}{ Perinatal outcome } & \multirow{2}{*}{ Total } & \multirow{2}{*}{ P-value } \\
\hline & Good $(n=981)$ & Adverse $(n=70)$ & & \\
\hline \multicolumn{5}{|c|}{ Fetal heart rate } \\
\hline Standard & 332 & 7 & 339 & \multirow{2}{*}{0.000033} \\
\hline Substandard & 649 & 63 & 712 & \\
\hline \multicolumn{5}{|c|}{ Cervical dilatation } \\
\hline Standard & 555 & 31 & 586 & \multirow{2}{*}{0.06} \\
\hline Substandard & 426 & 39 & 465 & \\
\hline \multicolumn{5}{|c|}{ Descent } \\
\hline Standard & 587 & 43 & 630 & \multirow{3}{*}{0.01} \\
\hline Substandard & 317 & 15 & 332 & \\
\hline Not Recorded & 77 & 12 & 89 & \\
\hline \multicolumn{5}{|c|}{ Maternal BP } \\
\hline Standard & 234 & 14 & 248 & \multirow{3}{*}{0.53} \\
\hline Substandard & 683 & 52 & 735 & \\
\hline Not Recorded & 64 & 4 & 68 & \\
\hline \multicolumn{5}{|c|}{ Maternal Body Temperature } \\
\hline Standard & 220 & 14 & 234 & \multirow{3}{*}{0.6545} \\
\hline Substandard & 679 & 52 & 731 & \\
\hline Not Recorded & 82 & 4 & 86 & \\
\hline \multicolumn{5}{|c|}{ Uterine contraction } \\
\hline Standard & 151 & 2 & 153 & \multirow{3}{*}{0.0056} \\
\hline Substandard & 789 & 67 & 856 & \\
\hline Not Recorded & 41 & 1 & 42 & \\
\hline \multicolumn{5}{|c|}{ Liquor state } \\
\hline Clear & 666 & 31 & 697 & \multirow{4}{*}{$\mathrm{P}<0.000001$} \\
\hline Meconium stained & 140 & 29 & 169 & \\
\hline Not recorded & 120 & 6 & 126 & \\
\hline Membrane intact & 55 & 4 & 59 & \\
\hline
\end{tabular}

Table 3: Association between quality of partogram recordings and perinatal outcomes.

Substandard records of uterine contraction is associated with adverse fetal outcomes ( $p$ value $=0.0056$, Chi-square 7.668).

Fetal outcomes seem not to be influenced by substandard recordings of maternal BP, PR and body temperature.
Meconium stained liquor seems to be associated with adverse fetal outcome as 29 out of 169 (17.2\%) of those with meconium stained liquor had adverse outcome compared to 31 out of $697(4.4 \%)$ of those with clear liquor ( $\mathrm{p}$-value $<0.001$, Chi-square 36.19 )

\begin{tabular}{|c|c|c|c|c|c|c|c|c|c|}
\hline \multirow{3}{*}{$\begin{array}{c}\text { Parameter of labor } \\
\text { Fetal heart rate }\end{array}$} & & \multicolumn{4}{|c|}{ Univariate Analysis } & \multicolumn{4}{|c|}{ Multivariate Analysis } \\
\hline & \multirow{3}{*}{\begin{tabular}{|c|} 
Number \\
339
\end{tabular}} & \multirow{4}{*}{$\begin{array}{c}\mathbf{O R} \\
4.604\end{array}$} & \multicolumn{2}{|c|}{$95 \% \mathrm{CI}$} & \multirow{4}{*}{$\begin{array}{c}\text { P-value } \\
0\end{array}$} & \multirow{4}{*}{$\begin{array}{c}\text { OR } \\
4.895\end{array}$} & \multicolumn{2}{|c|}{$95 \%$ CI } & \multirow{4}{*}{$\begin{array}{c}\text { P-values } \\
0.008\end{array}$} \\
\hline & & & \multirow{3}{*}{2.085} & \multirow{3}{*}{10.165} & & & \multirow{3}{*}{1.517} & \multirow{3}{*}{15.8} & \\
\hline Standard & & & & & & & & & \\
\hline Substandard and not recorded & 712 & & & & & & & & \\
\hline Descent & & \multirow{3}{*}{1.108} & \multirow{3}{*}{0.61} & \multirow{3}{*}{2.012} & \multirow{3}{*}{0.737} & \multirow{3}{*}{0.702} & \multirow{3}{*}{293} & \multirow{3}{*}{1.679} & \multirow{3}{*}{0.427} \\
\hline Standard & 394 & & & & & & & & \\
\hline Substandard and not recorded & 657 & & & & & & & & \\
\hline
\end{tabular}




\section{Women's Health Science Journal}

\begin{tabular}{|c|c|c|c|c|c|c|c|c|c|}
\hline State of Liquor & & \multirow{3}{*}{1.723} & \multirow{3}{*}{0.911} & \multirow{3}{*}{3.26} & \multirow{3}{*}{0.094} & \multirow{3}{*}{1.215} & \multirow{3}{*}{0.498} & \multirow{3}{*}{2.966} & \multirow{3}{*}{0.668} \\
\hline Standard & 697 & & & & & & & & \\
\hline Substandard and not recorded & 354 & & & & & & & & \\
\hline Cervical dilatation & & \multirow{3}{*}{1.647} & \multirow{3}{*}{1.011} & \multirow{3}{*}{2.683} & \multirow{3}{*}{0.045} & \multirow{3}{*}{1.784} & \multirow{3}{*}{0.762} & \multirow{3}{*}{4.179} & \multirow{3}{*}{0.182} \\
\hline Standard & 586 & & & & & & & & \\
\hline Substandard and not recorded & 465 & & & & & & & & \\
\hline Maternal BP & & \multirow{3}{*}{1.256} & \multirow{3}{*}{0.687} & \multirow{3}{*}{2.298} & \multirow{3}{*}{0.459} & \multirow{3}{*}{$1.02 \mathrm{E}+08$} & \multirow{3}{*}{0} & \multirow{3}{*}{. } & \multirow{3}{*}{0.999} \\
\hline Standard & 248 & & & & & & & & \\
\hline Substandard and not recorded & 803 & & & & & & & & \\
\hline Maternal Body Temperature & & \multirow{3}{*}{1.45} & \multirow{3}{*}{0.632} & \multirow{3}{*}{2.117} & \multirow{3}{*}{0.641} & \multirow{3}{*}{0} & \multirow{3}{*}{0} & \multirow{3}{*}{. } & \multirow{3}{*}{0.999} \\
\hline Standard & 234 & & & & & & & & \\
\hline Substandard and not recorded & 817 & & & & & & & & \\
\hline Uterine Contraction & & \multirow{3}{*}{6.186} & \multirow{3}{*}{1.5} & \multirow{3}{*}{25.508} & \multirow{3}{*}{0.012} & \multirow{3}{*}{1.45} & & & \\
\hline Standard & 153 & & & & & & 0.305 & 6.901 & 0.641 \\
\hline Substandard and not recorded & 898 & & & & & & & & \\
\hline
\end{tabular}

Table 4: Impact of quality of partogram recordings on fetal outcome.

OR- Odds Ratio. 95\% CI, ninety five percent confidence interval, BP- Blood Pressure.

The results show that substandard and lack of fetal heart rate monitoring is significantly associated with adverse fetal outcome and is about 4.6 times higher in those with substandard or lacking fetal heart rate monitoring (OR, 4.604, 95\% CI 2.09-10.17).

When fetal heart rate was adjusted for other factors (state of liquor, cervical dilatation assessment, maternal body temperature, Maternal Blood pressure, Descent and uterine contraction) still remained significantly associated though the association was weakened.

Uterine contractions had statistically significant association (OR 6.186, 95\% CI 1.50-25.51) with adverse fetal outcome. The association was lost when adjustment for other factors was done as shown in the (Table 4) above.

Maternal Blood pressure $(\mathrm{P}=0.045, \mathrm{OR}=1.647)$ and cervical dilatation $(\mathrm{P}=0.459, \mathrm{OR}=1.256)$ assessment had statistical marginal association with adverse fetal outcome and when adjusted for other factors the association was lost. Maternal body temperature, Descent and state of liquor had no statistically significant association with adverse fetal outcome (Table 5).

\begin{tabular}{|c|c|c|c|}
\hline \multirow{2}{*}{ Standard partograms } & \multicolumn{2}{|c|}{ Immediate Maternal outcomes } & \multirow{2}{*}{ P- value } \\
\cline { 2 - 3 } & Good (n=1029) & Adverse (n=22) & \multirow{2}{*}{0.8615} \\
\hline Substandard partograms & $83(8.1 \%)$ & $2(9.1 \%)$ & $20(90.9 \%)$ \\
\end{tabular}

Table 5: Association between quality of partogram and immediate maternal outcomes

Twenty-two women succumbed complications due to delivery. Two had cesarean hysterectomy, 11 got PPH, 5 had perineal tear, 1 got perineal hematoma, 2 had urinary bladder injury and 1 had bowel injury. Out of 22 who got complications, 20 (\%) had substandard partograms. There is no significant association between substandard partogram recordings and immediate adverse outcomes. $(\mathrm{P}$-value $=0.8615$, Chi square $=0.030)$.

\section{Discussion}

In this study it was found that all labors were monitored using partogram. Most of labor parameters had recorded at least once in the partogram. These findings show high proportion of partogram use in the hospital. Some partograms had no records for maternal pulse rate, descent of head, maternal body temperature and uterine contraction. These findings show that partogram use in this hospital is high as compared to other hospital which shows low proportion of partogram use during labor [11].

Despite the fact that partograms were used in all women in labor, majority (91.9\%) of them were judged substandard based on the recordings. This gives an impression that either labour monitoring in the hospital is 


\section{Women's Health Science Journal}

poor or there is inadequate recording of labour parameters in the partogram. This high proportion of substandard recordings of labour parameter were also found in other studies which assessed the partogram use during labor $[11,12]$.

Fetal heart and state of liquor are the two most important parameters which are used to detect fetal distress. Two thirds (67.7\%) of partograms in this study, had substandard recordings of fetal heart rate. This shows that based on these partogram recordings there was high probability of not diagnosing fetus that had distress on time. This study found that both Apgar score less than 7 at 5 minutes and still birth is significantly associated with substandard fetal heart monitoring during labor. The association between substandard fetal heart monitoring during labor and adverse fetal outcome was also found in a study done in Tanzania [13].

Uterine contractions and fetal presenting part descent had high proportion of substandard recordings in the reviewed partograms. Substandard recordings of these two parameters show weak association with adverse perinatal outcomes. Among the 70 new-born babies who had adverse fetal outcome 67 (95.7\%) had substandard recordings of uterine contraction. Though there is a high proportion of substandard record of uterine contraction but is somehow better when compared to other study done in Brazil, which showed that half of the women had no evaluation of uterine dynamics and $29 \%$ had no auscultation of fetal heart rate throughout labor [14].

In this study we found that all partograms had recordings of cervical dilatation though only $55.8 \%$ of them met the standard recordings. The cervical dilatation recordings in the partogram were found to have no significant association with fetal outcome. These findings concur with that found in the study done in Tanzania [13].

The condition of new-born baby is recorded in the partogram. Eighty nine new-borns were admitted in neonatal ward for special care. Among those admitted majority (59.5\%) were due to Apgar score less than 7 at 5 minute but also abnormal breathing and meconium aspiration contributed 9 and 8newborns respectively. There were no details in the partogram concerning those babies who had abnormal breathing.

There were 70 adverse perinatal outcomes (53 low Apgar scores and 17 stillbirth) among the reviewed partograms. Cord around the neck and thick meconium was the only detail found in some partograms of stillbirth. The cesarean section rate of the reviewed partograms were $18.6 \%$, all were emergency. Only 4 women who had vaginal birth had immediate maternal adverse outcome compared with 18 who had caesarean birth. Majority of the partograms of those who succumbed complications were judged to be substandard. There is no significant association found between substandard partogram and immediate maternal adverse outcomes.

\section{Strength of the Study}

Large sample size with immediate review of partograms after birth. The use of independent reviewer of the partograms using a standard checklist.

\section{Limitation of the Study}

Lack of observation of actual midwives performance during labor management, lack of details of stillbirths and follow-up to know the outcomes of those new-born with low Apgar scores admitted to neonatal unit.

\section{Conclusion}

In this study it was found out that partogram were used in all women in labor at MNH. Though there were high prevalence of partogram use but most of them had substandard recordings of parameters of labor. Substandard fetal heart rate monitoring, uterine contractions and meconium stained liquor are significantly associated with Apgar score less than 7 at 5 minute and stillbirth. This means that association between quality of partogram recordings and perinatal outcomes does exist. There is no association between quality of partogram recordings and immediate maternal outcomes.

\section{Competing Interests}

All authors declare that there is no conflict of interest regarding the publication of this paper.

\section{Funding}

This study was funded as part of master's program by Tanzania Ministry of Health through master studies scholarship.

\section{Authors' Contributions}

The first and second author were involved in study design, data collection, data analysis and manuscript writing. The last author was involved in study design, data analysis and manuscript writing. All authors revised this manuscript and approved it for submission. 


\section{Women's Health Science Journal}

\section{Acknowledgements}

We acknowledge and appreciate all women who gave birth at MNH and midwives working at the MNH labor ward during the study time.

\section{References}

1. (1994) World Health Organization partograph in management of labour. World Health Organization Maternal Health and Safe Motherhood Programme. Lancet 343(8910): 1399-1404.

2. Walraven GEL (1994) WHO partograph. The Lancet 344(8922): 617.

3. Dujardin B, De Schampheleire I, Sene H, Ndiaye F (1992) Value of the alert and action lines on the partogram. Lancet 339(8805): 1336-1338.

4. Mathibe Neke JM, Lebeko FL, Motupa B (2013) The partograph: A labour management tool or a midwifery record? International Journal of Nursing and Midwifery 5(8): 145-153.

5. United Nations Inter-Group for Child Mortality Estimation (2015) Levels and trends in child mortality: Report 2015. UNICEF WHO World Bank Gr United Nations.

6. Susannah B, Leisher H, Lawn JE, Kinney M V, Kuo NT, et al. (2016) Stillbirths: Investment in ending preventable stillbirths by 2030 will yield multiple returns and help achieve multiple Sustainable Development Goals. Lancet 1-5.

7. Kidanto HL, Massawe SN, Nystrom L, Lindmark G (2006) Analysis of perinatal mortality at a teaching hospital in Dar es Salaam, Tanzania, 1999-2003. Afr J Reprod Health 10(2): 72-80.
8. Kidanto HL, Mogren I, van Roosmalen J, Thomas AN, Massawe SN, et al. (2009) Introduction of a qualitative perinatal audit at Muhimbili national hospital, Dar es Salaam, Tanzania. BMC Pregnancy Childbirth 9: 45 .

9. Lansky S, Franca E, Cesar CC, Monteiro Neto LC, Leal $M$ do C (2006) [Perinatal deaths and childbirth healthcare evaluation in maternity hospitals of the Brazilian Unified Health System in Belo Horizonte, Minas Gerais, Brazil, 1999]. Cad Saude Publica 22(1): 117-130.

10. Maaløe N, Housseine N, Bygbjerg IC, Meguid T, Khamis RS, et al. (2016) Stillbirths and quality of care during labour at the low resource referral hospital of Zanzibar: A case-control study. BMC Pregnancy Childbirth 16(1): 351.

11. Ogwang S, Karyabakabo Z, Rutebemberwa E (2009) Assessment of partogram use during labour in Rujumbura Health Sub District, Rukungiri District, Uganda. Afr Heal Sci 9(1): S27-34.

12. Azandegbe N, Testa J, Makoutode M (2004) [Assessment of partogram utilisation in Benin]. Sante 14(4): 251-255.

13. Nyamtema a S, Urassa DP, Massawe S, Massawe a, Lindmark G, et al. (2008) Partogram use in the Dar es Salaam perinatal care study. Int J Gynaecol Obstet 100(1): 37-40.

14. Giglio MRP, Franca E, Lamounier JA (2011) Evaluation of the quality of care for normal delivery. Revista Brasileira de Ginecologia e Obstetrícia 33(10): 297-304.

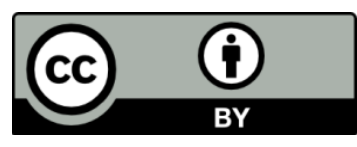

